\section{Review Article}

Check for updates

\title{
Multi Modality Imaging Features of Cardiac Myxoma
}

\author{
Brylie J. McAllister $\mathbb{D}$, BSc, MBBS
}

OPEN ACCESS

Received: Feb 24, 2020

Revised: Apr 10, 2020

Accepted: Apr 24, 2020

Address for Correspondence:

Brylie J. McAllister, BSc, MBBS

Department of Radiology, Gold Coast

University Hospital, 1 Hospital Blvd, Southport,

QLD, 4215, Australia.

E-mail: brylie.mcallister@gmail.com

Copyright $(2020$ Korean Society of

Echocardiography

This is an Open Access article distributed

under the terms of the Creative Commons

Attribution Non-Commercial License (https://

creativecommons.org/licenses/by-nc/4.0/)

which permits unrestricted non-commercial

use, distribution, and reproduction in any

medium, provided the original work is properly cited.

ORCID iDs

Brylie J. McAllister (iD)

https://orcid.org/0000-0002-2468-3016

Conflict of Interest

The authors have no financial conflicts of interest.

\author{
Department of Radiology, Gold Coast University Hospital, Southport, QLD, Australia
}

\section{ABSTRACT}

Primary cardiac neoplasms are rare entities of which approximately $75 \%$ are benign and the remaining 25\% malignant. Myxomas are the most common benign primary cardiac tumor $(30 \%)$ and most commonly arise in the left atrium from the interatrial septum at the fossa ovalis. However, they also can originate in any cardiac chamber. Clinical presentation and patient symptomatology are determined by size, location, and mobility of the myxoma. This review will discuss the clinical presentation, natural history, pathology, and multimodality imaging features of cardiac myxomas.

Keywords: Cardiac myxoma; Cardiac mass; Multimodality imaging

\section{INTRODUCTION}

Primary cardiac neoplasms are rare entities (0.001\%-0.3\% of autopsies) of which approximately $75 \%$ are benign and the remaining $25 \%$ malignant. ${ }^{1)}$

Myxomas are the most common benign primary cardiac tumor (30\%). Other less common benign entities include papillary fibroelastoma, fibroma, rhabdomyoma, haemangioma, lipoma and paraganglioma. On the malignant spectrum, the most common is sarcoma (75\%), followed by secondary metastases. ${ }^{1}$ Primary cardiac lymphoma and pericardial mesothelioma are both rare entities.

In patients with myxoma, there is a broad range in the age of presentation (11-82 years). Most present in adulthood (mean 50 years), and there is a female predominance. ${ }^{2)}$ Ninety percent of cases are sporadic. The remaining $10 \%$ are associated with Carney syndrome where the patients happen to be younger (mean 24 years) and usually men (66\% vs $24 \%$ sporadic). ${ }^{3)}$ Cardiac myxoma is one of the major diagnostic criteria for diagnosis of this complex, requiring two or more major diagnostic criteria and/or by identification of a heterozygous germline pathologic variant in PRKAR1A on molecular genetic testing (Table 1). ${ }^{4)}$

Myxomas most commonly arise in the left atrium (LA) from the interatrial septum at the fossa ovalis, however they may originate in any cardiac chamber (75\% LA, 20\% right atrium [RA], rarely right ventricle or left ventricle). Less common locations include the posterior atrial 
Table 1. Diagnostic criteria for carney complex

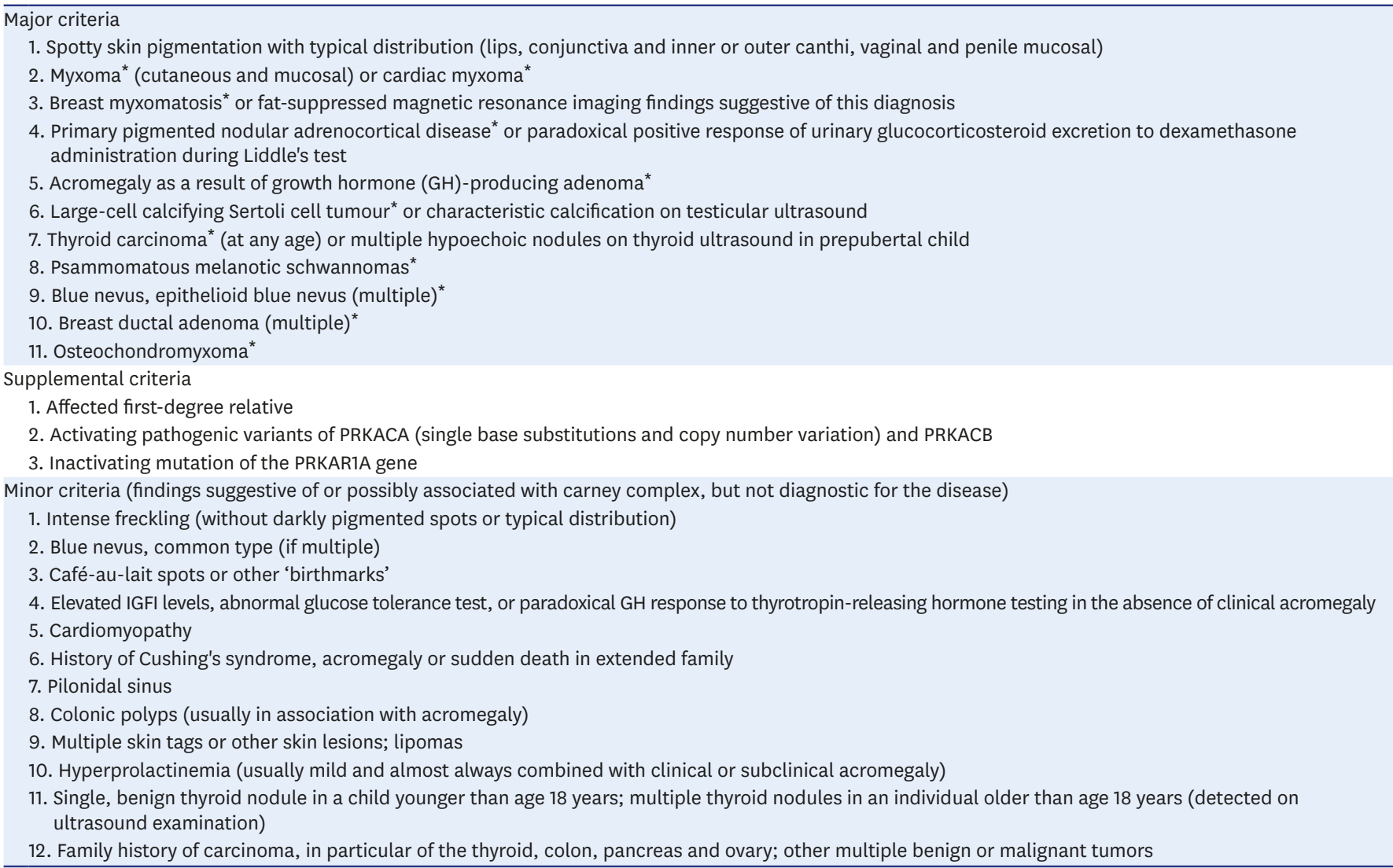

To make the diagnosis of carney complex, a patient must either (1) exhibit two of the major criteria confirmed by histology, imaging or biochemical testing or (2) meet one major criterion and one supplemental one. ${ }^{11)}$

*With histologic confirmation.

wall, anterior atrial wall, and atrial appendage. Very rarely they may occur on the heart valves. Multiple tumors and atypical locations are more frequent in cases of familial myxoma. ${ }^{5}$

This paper will review the clinical presentation, natural history, and pathology of cardiac myxomas and provide an overview of imaging features.

\section{CLINICAL PRESENTATION}

Clinical presentation and patient symptomatology are determined by the size, location and mobility of the myxoma.

Most patients present with one or more of the triad of (1) embolization, (2) intracardiac obstruction, and (3) constitutional symptoms, ${ }^{5}$ ) therefore, presentation may mimic infective endocarditis.

The most common symptom relates to valvular obstruction and occurs in approximately $50 \%$ of patients. Systolic murmurs may occur if there is interference with closure of the atrioventricular $(\mathrm{AV})$ valves or ventricular outflow tract narrowing; diastolic murmurs are due to obstructed ventricular filling. Valvular obstruction may also result in heart failure, syncope or sudden death. 
Polypoid and extensively myxoid lesions, or those that are irregular are more likely to form surface thrombi with subsequent embolization, the second most common manifestation of myxoma occurring in 30\%-40\% of patients. ${ }^{2}$ Embolization may result in central nervous system symptoms including transient ischaemic attacks, stroke or seizure; visceral infarction and pulmonary embolism may also occur.

Constitutional symptoms including weight loss, malaise, fever, arthralgia and myalgia are due to tumor production of IL-6. There is a positive correlation between tumor size and IL-6 production levels; in addition, the higher the IL-6 levels, the more intense the constitutional symtpoms. $\left.{ }^{6}\right)$

Twenty percent of cases are asymptomatic. ${ }^{2)}$

\section{NATURAL HISTORY}

Because myxomas are usually excised following diagnosis, their rate of growth is generally unknown. Previous case reports have demonstrated lesion stability on serial imaging up to a period of 15 years, ${ }^{7}$ with growth rates of up to $1.36 \times 0.27 \mathrm{~cm} /$ month. $^{8)}$

\section{HISTOPATHOLOGY}

Myxomas are neoplasms of endocardial origin, projecting into the cardiac chamber.

They are generally polypoid, often pedunculated, rarely sessile, and round or oval with a smooth or gently lobulated appearance. The less common villus type has friable, frond like contours, which have a greater propensity for fragmentation and subsequent embolization. ${ }^{9)}$ Most are 5-6 cm, and can range from 1-15 cm in diameter (Figure 1). ${ }^{5)}$

Myxomas are composed of stellate or globular myxoma cells with abundant eosinophilic cystoplasm, indistinct cell borders, oval nucleus with open chromatin and indistinct nuclei (Figure 2). Myxoma cells form complex structures including rings, syncytia, and cords that are typically infiltrated by lymphocytes and macrophages. ${ }^{2)}$

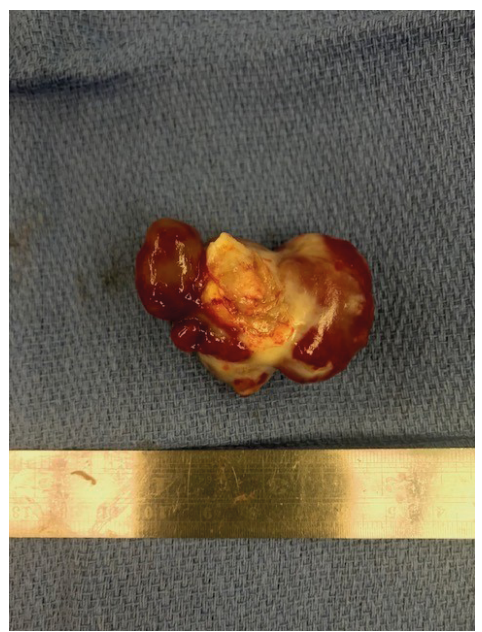

Figure 1. Excised left atrial cardiac myxoma with a pedicle. 


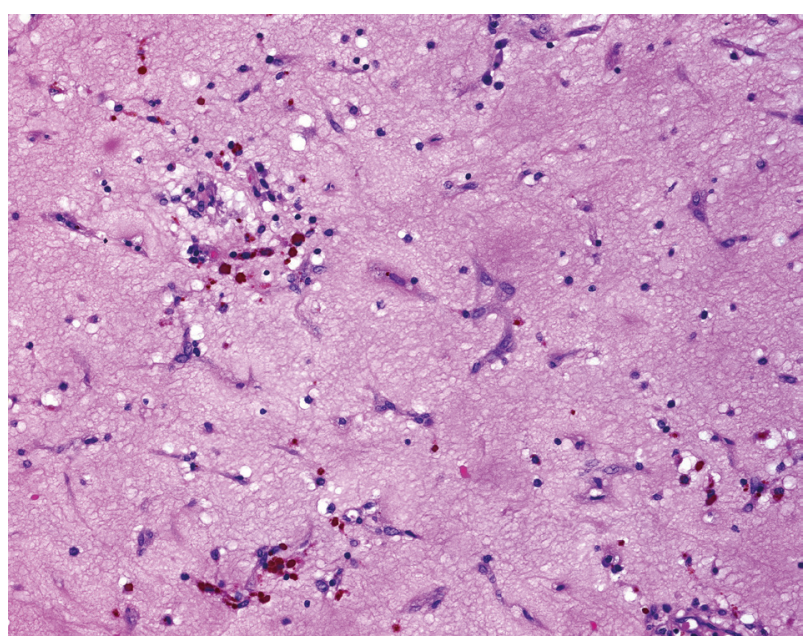

Figure 2. H\&E stain: stellate cells in abundant myxoid matrix (image used with permission of WebPathology)

Histologically, myxomas may exhibit variable fibrosis (41\%), calcification (20\%), GamnaGandy bodies (17\%), ossification (8\%), extramedullary hematopoesis (7\%), mucin forming glands $(3 \%)$, atypia $(3 \%)$, and/or thymic rests $(1 \%) .{ }^{10)}$

\section{IMAGING FEATURES}

\section{Echocardiogram}

Echocardiogram is typically the primary imaging modality with high sensitivity and specificity, allowing to assess the size, morphology, attachment site, mobility and haemodynamic consequences of the tumor.

Trans-oesophageal echocardiogram (TOE) is superior to trans-thoracic echocardiogram (TTE), providing more detailed evaluation, ${ }^{2)}$ particularly with smaller lesions.

Myxomas manifest as spherical masses attached to the endocardial surface, with occasional internal hypoechoic areas, speckled echogenic foci (Figure 3A), and frond-like surface projections. ${ }^{2)}$ Areas of calcification are typically echogenic (Figure 4A); Doppler images may demonstrate internal flow (Figure 5B), particularly in those with capillary-like channels that communicate with the surface of the myxoma. ${ }^{5}$

Echocardiogram contrast agents may be useful to assess vascularity of the tumor, with highly vascular lesions demonstrating hyper-enhancement. Real-time three dimensional (3D) echocardiogram allows for a volumetric assessment of a mass over a linear measurement as it is obtained with 2-dimensional imaging. With the cropping techniques available with 3D, various aspects of the mass can be better visualized including point of attachment, homogeneity, vascularity, and calcification. ${ }^{11}$

Limiting factors of echocardiogram include a narrower field of view compared to computed tomography (CT) and magnetic resonance imaging (MRI), a poor acoustic window, and artifacts that may be misinterpreted as pathology. 

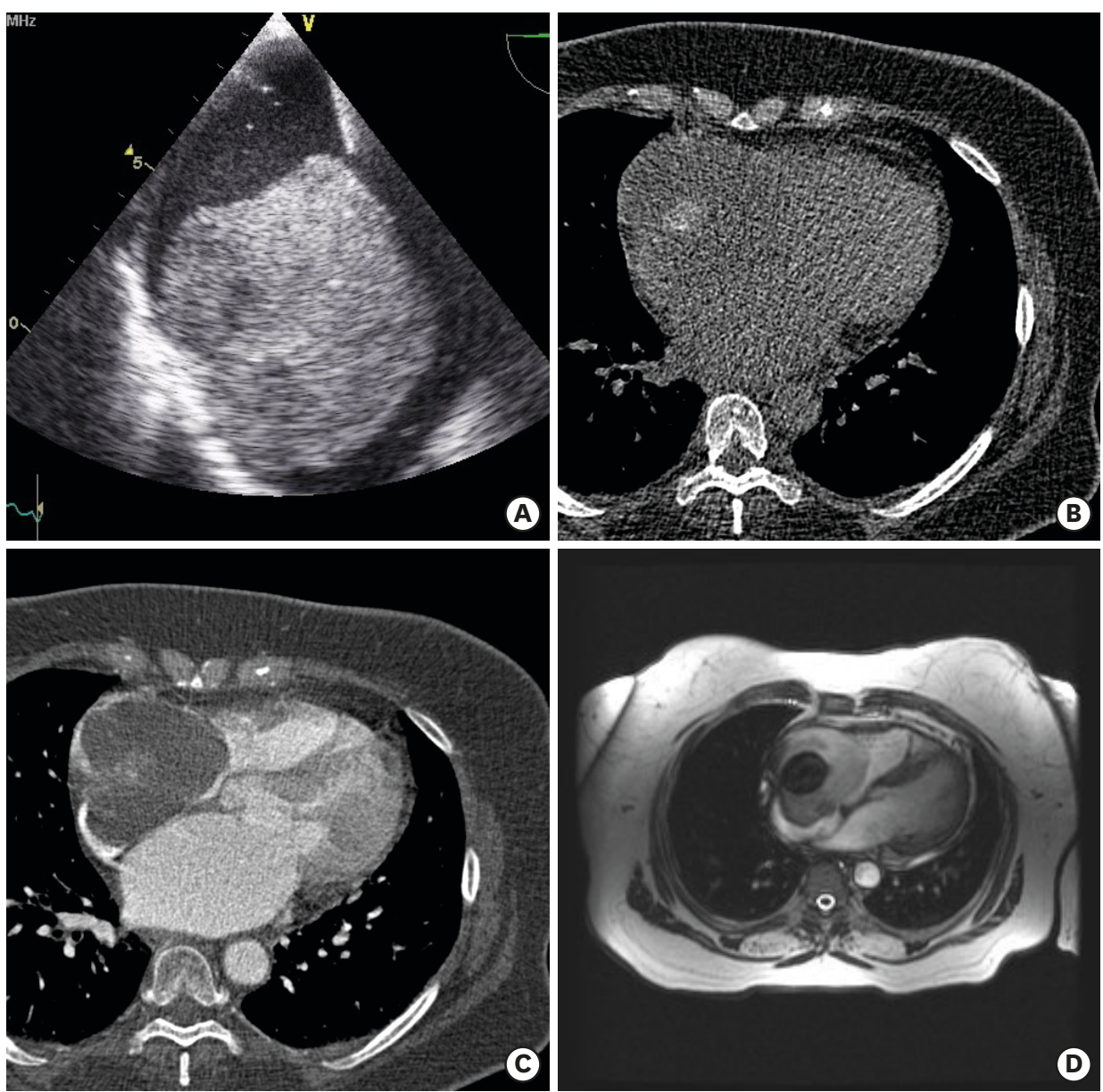

Figure 3. (A) TTE demonstrates the large right atrial mass with small, specked echogenic foci, prolapsing in to the right ventricle and bulging the inter atrial septum to the left. (B) Right atrial myxoma is slightly lower attenuation than blood pool, and is heterogenous in density secondary to calcification; (C) post contrast CT better demonstrates the massive myxoma attached by a pedicle to the posterior wall of the right atrium. The mass occupies almost the entire chamber. There is minimal contrast enhancement; (D) corresponding T2 MRI demonstrating central low signal secondary to calcification. The remainder of the mass is of high signal intensity; CT: computed tomography, MRI: magnetic resonance imaging, TTE: trans-thoracic echocardiogram.
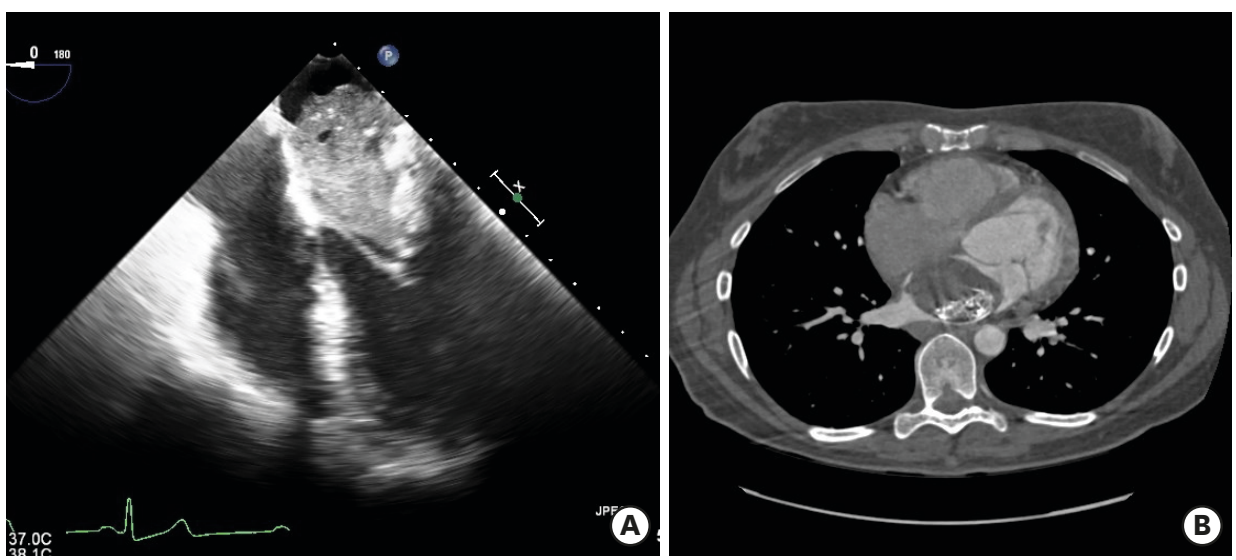

Figure 4. (A) TOE demonstrating hyperechoic foci corresponding to the calcification on CT. There is mild prolapse through the mitral valve leaflets during diastole. (B) Post contrast CT chest demonstrating a large left atrial myxoma attached to the interatrial septum at the level of the fossa ovalis. High density in the posterior aspect of the mass is secondary to calcification. CT: computed tomography, TOE: trans-oesophageal echocardiogram. 

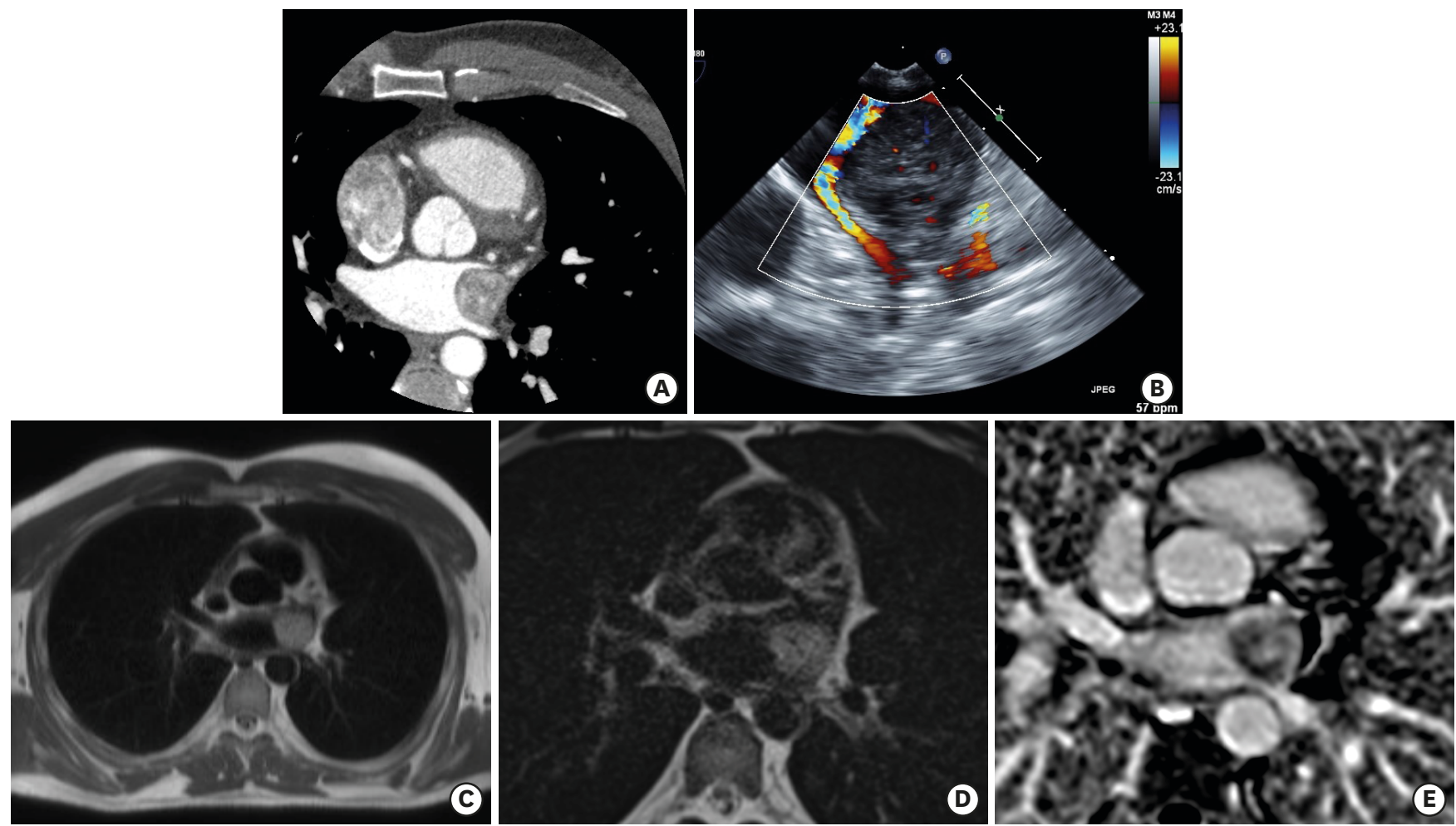

Figure 5. (A) Cardiac CT with a heterogenous mass at the base of the left atrial appendage with foci of contrast density, seen with capillary-like channels that communicate with the surface of the myxoma; (B) TOE Doppler showing small areas of flow within the myxoma; (C) T1 and (D) black blood MRI images, the mass is hyperintense; (E) central contrast enhancement on LGE helps to distinguish this tumor from thrombus. CT: computed tomography, LGE: late gadolinium enhancement, MRI: magnetic resonance imaging, TOE: trans-oesophageal echocardiogram.
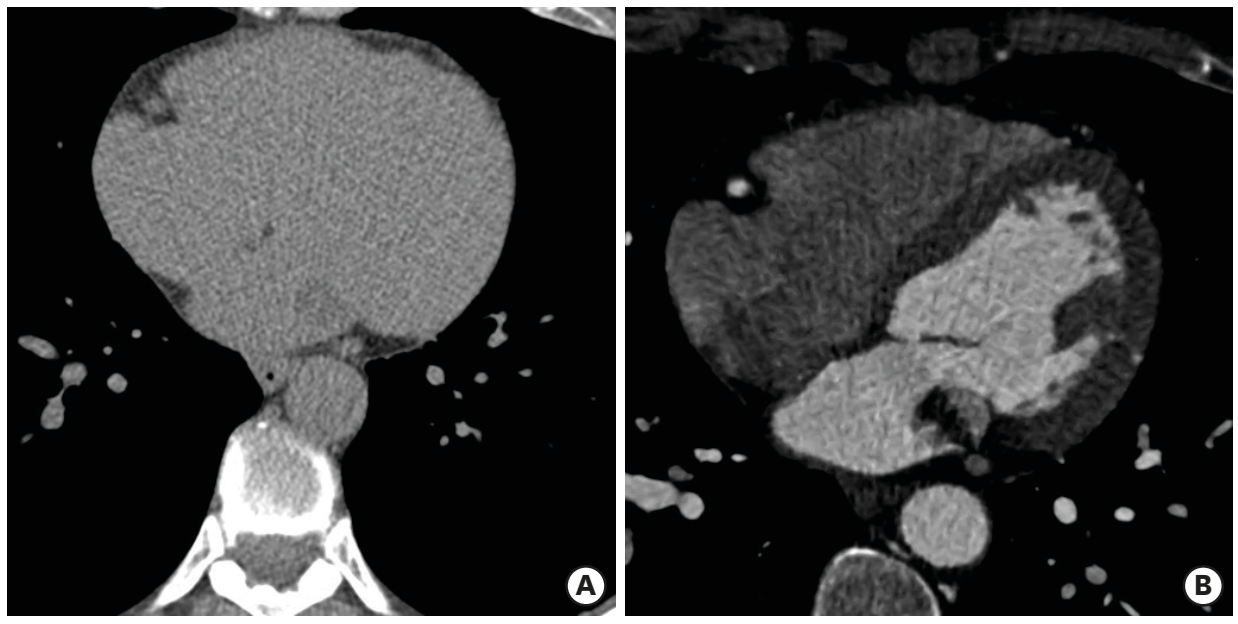

Figure 6. (A) Non contrast cardiac computed tomography with myxoma arising from the posterior wall of the left atrium, contacting the posterior mitral valve leaflet. Note the density of the mass is lower attenuation than blood pool. (B) Contrast enhanced image performed in diastole. The mitral valve is open, there is no prolapse of the mass in to the left ventricle. There was mild enhancement.

\section{Computed tomography}

On non-contrast CT, tumor attenuation is typically lower than non-opacified blood (Figure 6A). Myxomas often appear heterogenous due to hemorrhage, calcification/ossification (Figures 3B, 3C, and 4), necrosis, cyst formation or fibrosis. Tumors may visibly enhance post contrast administration, but typically enhancement is less evident than with MRI 


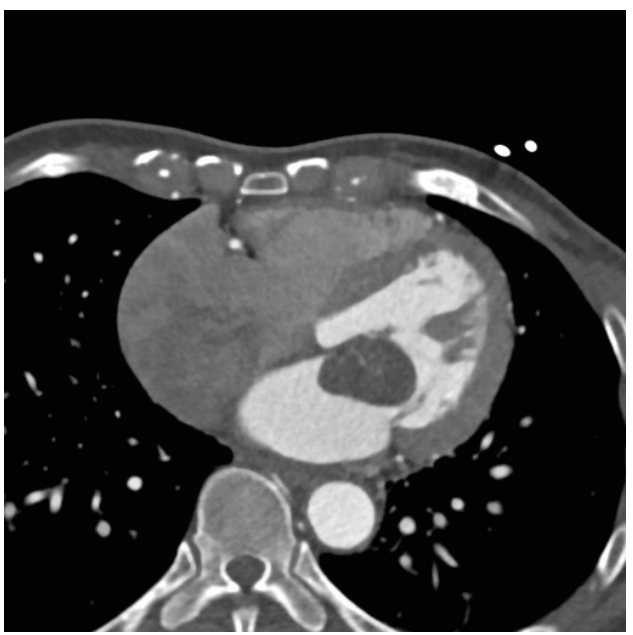

Figure 7. Cardiac computed tomography. Left atrial myxoma contacting the anterior leaflet of the mitral valve, prolapsing in to the left ventricle during early diastole.

Table 2. MRI protocol for suspected cardiac mass

MRI protocol
Localiser images
4-chamber/3-chamber/2-chamber long axis cine SSFP sequences
Cine SSFP images in 2 planes oriented perpendicular to the lesion
T1/T2/T2FS black blood imaging sequences in 2 planes oriented perpendicular to the lesion
Short axis LV stack cine SSFP (optional)
First pass perfusion imaging in 2 perpendicular planes through the lesion
LGE in at least 2 planes oriented perpendicular to the lesion

FS: fat saturated. LGE: late gadolinium enhancement. LV: left ventricle. SSFP: steady state free procession.

(Figure 6B) and can be difficult to appreciate due to surrounding high contrast blood pool. Dual energy CT with mean iodine concentration is an accurate approach for defining whether a cardiac mass visibly enhances. ${ }^{12}$

Functional retrospective cardiac CT is able to demonstrate tumor mobility (Figure 7).

\section{Magnetic resonance imaging}

MRI is the modality of choice for evaluation and assessment of cardiac tumors due to its superior tissue characterization and ability to aide in differential diagnosis. With cine and phase contrast sequences, one is able to assess the functional impact of the mass. See Table 2 for a standard MRI protocol for patients with suspected cardiac myxomas. ${ }^{12)}$

Myxomas can be substantially variable in appearance and of heterogenous signal intensity on MRI owing to myxoid tissue, fibrous tissue, blood and calcification.

T1 and T2 weighted double inversion recovery fast spin echo sequences with nulling of the blood pool in relation to the myocardium ("black blood") and black blood fat saturated T2 weighted sequences are able to assist tissue characterization. ${ }^{9)}$ On T1 the tumors are usually isointense to myocardium (intermediate signal intensity), and occasionally hyperintense.

Myxomatous components are low on T1, high on T2. ${ }^{2)}$ Low signal on both T1 and T2 may be due to calcification (Figure 3D); haemorrhage within the tumor is variable in signal, depending on age. 
Late gadolinium enhancement sequences performed 10-15 minutes after contrast administration are helpful to differentiate these tumors from thrombus, particularly if they arise in the LA appendage (Figure 5E). Enhancement is typically heterogenous, and areas of enhancement have been shown to correspond with regions rich in myxomatous tissue and focal inflammation. ${ }^{9)}$ There may be non-enhancing areas secondary to internal cysts or necrosis. First pass perfusion studies may demonstrate mild heterogenous enhancement.

An additional sequence that can aide in the differentiation between myxoma and thrombus is inversion time (TI). TI varies between patients but is typically approximately $300 \mathrm{~ms}$, depending on the cardiac output and time after contrast injection. ${ }^{13)}$ Compared with normal myocardium, Pazos-López et al..$^{14)}$ found the majority of thrombi $(94 \%)$ tend to be hyperintense/isointense with short TI (150 ms), and hypointense with long TI (500 ms); tumors rarely follow this pattern of signal $(2 \%)$.

Cine steady state free procession images are useful in the functional assessment of the myxoma, as mobile lesions can prolapse through the AV valve during diastole.

If there is associated mitral valve obstruction, features such as LA enlargement and pulmonary venous hypertension with pulmonary vascular redistribution and pulmonary oedema may be seen on radiographs, CT and MRI.

\section{CONCLUSION}

Myxomas are the most frequently diagnosed primary cardiac tumor. They have characteristic imaging features that may frequently suggest the diagnosis and aid in differentiating these lesions from other intracardiac masses, facilitating the choice of appropriate therapeutic management.

Whilst echocardiogram can readily assess cardiac myxomas and suggest the diagnosis in many cases, further imaging with contrast enhanced CT and MRI is beneficial in providing greater anatomical detail for surgical planning. MRI is most useful for differential diagnoses, the most common of which include metastases and thrombus, as well as other primary benign and malignant neoplasms, and valvular vegetations. Once diagnosed, treatment consists of urgent surgical excision due to potential life threatening sequelae including embolic complications and sudden cardiac death. Surgical excision has excellent long-term prognosis and low risk of recurrence. ${ }^{2)}$

\section{REFERENCES}

1. Reardon MJ, DeFelice CA, Sheinbaum R, Baldwin JC. Cardiac autotransplant for surgical treatment of a malignant neoplasm. Ann Thorac Surg 1999;67:1793-5. PUBMED | CROSSREF

2. Grebenc ML, Rosado de Christenson ML, Burke AP, Green CE, Galvin JR. Primary cardiac and pericardial neoplasms: radiologic-pathologic correlation. Radiographics 2000;20:1073-103. PUBMED | CROSSREF

3. Carney JA. Differences between nonfamilial and familial cardiac myxoma. Am J Surg Pathol 1985;9:53-5. PUBMED | CROSSREF

4. Stratakis CA, Raygada M. Carney Complex. 2003 Feb 5 [updated 2018 Aug 16]. In: Adam MP, Ardinger HH, Pagon RA, et al., editors. GeneReviews ${ }^{\circledR}$ [Internet]. Seattle (WA): University of Washington, Seattle; 1993-2020. Available from: https://www.ncbi.nlm.nih.gov/books/NBK1286/ 
5. Reynen K. Cardiac myxomas. N Engl J Med 1995;333:1610-7. PUBMED | CROSSREF

6. Mendoza CE, Rosado MF, Bernal L. The role of interleukin-6 in cases of cardiac myxoma. Clinical features, immunologic abnormalities, and a possible role in recurrence. Tex Heart Inst J 2001;28:3-7. PUBMED

7. Kay JF, Chow WH. Long-term survival of quiescent left atrial myxoma in an elderly patient. Am J Geriatr Cardiol 2002;11:165-8. PUBMED | CROSSREF

8. Karlof E, Salzberg SP, Anyanwu AC, Steinbock B, Filsoufi F. How fast does an atrial myxoma grow? Ann Thorac Surg 2006;82:1510-2. PUBMED | CROSSREF

9. Abbas A, Garfath-Cox KA, Brown IW, Shambrook JS, Peebles CR, Harden SP. Cardiac MR assessment of cardiac myxomas. Br J Radiol 2015;88:20140599. PUBMED | CROSSREF

10. Pernick N. Myxoma [Internet]. PathologyOutlines.com website. Available from: http://www. pathologyoutlines.com/topic/hearttumormyxoma.html

11. Mankad R, Herrmann J. Cardiac tumors: echo assessment. Echo Res Pract 2016;3:R65-77. PUBMED | CROSSREF

12. Hong YJ, Han K, et al. Quantitative analysis of a whole cardiac mass using dual-energy computed tomography: comparison with conventional computed tomography and magnetic resonance imaging. $\mathrm{Sci}$ Rep 2018;8:15334. PUBMED | CROSSREF

13. O'Donnell DH, Abbara S, Chaithiraphan V, et al. Cardiac tumors: optimal cardiac MR sequences and spectrum of imaging appearances. AJR Am J Roentgenol 2009;193:377-87. PUBMED | CROSSREF

14. Pazos-López P, Pozo E, Siqueira ME, et al. Value of CMR for the differential diagnosis of cardiac masses. JACC Cardiovasc Imaging 2014;7:896-905. PUBMED | CROSSREF 